\title{
ADAR2 functions as a tumor suppressor via editing IGFBP7 in esophageal squamous cell carcinoma
}

\author{
YUAN-BIN CHEN ${ }^{1,2}$, XIAO-YU LIAO ${ }^{1,3}$, JIANG-BO ZHANG ${ }^{1}$, FANG WANG ${ }^{1}$, HAI-DE QIN $^{4}$, \\ LANJUN ZHANG $^{1}$, YIN YAO SHUGART ${ }^{4}$, YI-XIN ZENG ${ }^{1}$ and WEI-HUA JIA ${ }^{1}$ \\ ${ }^{1}$ State Key Laboratory of Oncology in South China, Collaborative Innovation Center for Cancer Medicine, \\ Sun Yat-sen University Cancer Center, Guangzhou, Guangdong 510060; ${ }^{2}$ Central Laboratory, \\ Affiliated Hospital of Qingdao University, Qingdao, Shandong 266000; ${ }^{3}$ Department of Endocrinology, \\ Xinqiao Hospital, Third Military Medical University, Chongqing 400037, P.R. China; \\ ${ }^{4}$ Unit on Statistical Genomics, Division of Intramural Research Programs, \\ National Institute of Mental Health, National Institutes of Health, Bethesda, MD 20892, USA
}

Received October 16, 2016; Accepted November 28, 2016

DOI: 10.3892/ijo.2016.3823

\begin{abstract}
Esophageal squamous cell carcinoma (ESCC), one of the most aggressive cancers, is characterized by heterogeneous genetic and epigenetic changes. Recently, A-to-I RNA editing, catalyzed by adenosine deaminases acting on RNA (ADARs), was found to be aberrantly regulated during tumorigenesis. We previously reported that ADAR2 was downregulated in ESCC but its role was unclear. Thus, we report here that overexpression of ADAR2 can induce apoptosis in ESCC cell lines and inhibit tumor growth in vitro and in vivo. ADAR2 knockdown inhibited apoptosis in ADAR2 highly expressing tumor cells. RNA-seq assay showed that ADAR2, not ADAR1 or active-site-mutated ADAR2, could edit insulin-like growth factor binding protein 7 (IGFBP7) mRNA in ESCC. IGFBP7 knockdown or ADAR2 catalytic activity destruction abolished the pro-apoptotic function of ADAR2. Mechanistically, RNA editing may stabilize IGFBP7 protein by changing the protease recognition site of matriptase and this is essential for IGFBP7 to induce apoptosis. Western blotting revealed that ADAR2 overexpression could induce IGFBP7-dependent inhibition of Akt signaling. Thus, our data indicate that ADAR2 suppresses tumor growth and induces apoptosis by editing and stabilizing IGFBP7 in ESCC, and this may represent a novel therapeutic target for treating ESCC.
\end{abstract}

Correspondence to: Dr Wei-Hua Jia, State Key Laboratory of Oncology in South China, Sun Yat-sen University Cancer Center, Building 2, Room 903, 651 Dongfeng Road East, Guangzhou, Guangdong 510060, P.R. China

E-mail: jiawh@sysucc.org.cn

Key words: ADAR2, esophageal neoplasms, IGFBP7, RNA editing, RNA sequencing

\section{Introduction}

Esophageal squamous cell carcinoma (ESCC), a heterogeneous tumor containing various genetic and epigenetic changes, is a leading cause of cancer death worldwide. Unlike esophageal adenoma carcinoma (EAC), which is common in some developed countries, ESCC is most prevalent in underdeveloped regions (1). Despite recent therapeutic advances, ESCC outcomes are unsatisfactory; only $15-25 \%$ of ESCC patients survive 5 years (2). To investigate the driving mutation behind ESCC and find a potential therapeutic target, recent genomics studies using high-throughput sequencing have been done by our group and others. However, these studies yielded few mutated genes with frequencies as high as already known mutated genes such as TP53, NOTCH1 and NFE2L2 (3-7).

RNA editing is an epigenetic process of post-transcriptional modification. The most common human RNA editing is the conversion of adenosine (A) to inosine (I), which is catalyzed by adenosine deaminases acting on the RNA protein family (ADARs). Three members of the ADAR gene family exist, ADAR1, ADAR2 (or ADARB1), and ADAR3. ADAR1 and ADAR2 are ubiquitously expressed, whereas ADAR3 is localized to the brain (8). Because I is recognized as guanosine $(\mathrm{G})$ by translation machinery, ADARs may recode the transcriptome. If the editing event occurs in the coding region, the protein sequence, structure, and function can be altered.

Since the first report of altered RNA editing in brain cancer in 2001 (9), more studies have supported the idea that misregulation of ADARs are key to tumorigenesis. ADAR1 and ADAR2 can edit many similar sites, but other sites are clearly specific for one or the other (10), so dysregulation of either can uniquely affect tumorigenesis. In fact, ADAR1 appears to act as an oncogene in cancers (11-14), including ESCC (15), whereas ADAR2 was reported to be a suppressor of brain tumors (16) and hepatocellular carcinoma (14). Our previous genomic study indicated that a gene amplification 
region containing microRNA, miR-4707-5p, could aberrantly suppress ADAR2 in ESCC (3), but the function of ADAR2 in ESCC has historically been poorly understood.

IGFBP7, or IGFBP-related protein 1 (IGFBP-rP1), has been shown to bind to (and interfere with the activation of) IGF1R, block downstream Akt signaling, and induce apoptosis (17). Although IGFBP7 is reported to be an editing target $(18,19)$, the relationship between ADAR2 and IGFBP7 is not clear in ESCC. Thus, we report that IGFBP7 is a novel target gene of ADAR2 in ESCCs, as evidenced by whole transcriptome data and direct sequencing. We also describe the antitumor role and mechanism of ADAR2 in ESCC in vitro and in vivo.

\section{Materials and methods}

Cell lines. An ESCC EC109 cell line was obtained from the Cell Bank of the Chinese Academy of Sciences (Shanghai, China). The Japanese ESCC KYSE30 cell line was generously provided by Dr Guan Xinyuan from Sun Yat-sen University Cancer Center (SYSUCC). These two cell lines were maintained in RPMI-1640. The HEK293T cell line (used for lentivirus packaging) was obtained from SYSUCC and maintained in DMEM. We added $10 \%$ fetal bovine serum and $1 \mathrm{mM}$ penicillin/streptomycin to the media.

Vector construction and transfection assay. For siRNA transfection, Lipofectamine RNAiMAX reagent (Invitrogen) was used according to kit directions. siRNAs were purchased from RiboBio (Guangzhou, China). For gene transient transfection, coding sequences were cloned into pcDNA3.1, and the plasmids were transfected with Lipofectamine 2000 (Invitrogen). For gene stable transfection, pLVX-IRES-Puro lentiviral expression vector (Clontech) was used according to the manufacturer's instructions.

Cell growth and clone formation. Cell growth was measured by MTT assay as previously reported (20), using $2 \times 10^{3}$ cells seeded into each well of a 96-well plate. A clone formation assay was carried out as previously reported (15), using $1 \times 10^{3}$ cells seeded into each well of a 24-well plate.

Apoptosis assay. Apoptosis was measured using an Annexin V-FITC and PI staining kit (Bestbio, Shanghai, China) according to the manufacturer's protocol. Briefly, cells were collected, washed and resuspended in $400 \mu \mathrm{l}$ binding buffer containing $5 \mu \mathrm{l}$ Annexin V-FITC and $10 \mu \mathrm{l}$ PI. After incubation, samples were assessed with flow cytometry. We summed percents of early apoptotic cells (Annexin V-positive, PI-negative) and late apoptotic or necrotic cells (Annexin V/ PI-double-positive) to obtain percent apoptotic cells.

Animal tumorigenicity experiments. Animal experiments were approved by the Institutional Animal Care and Use Committee at SYSUCC. Five-week old female BALB/C-nu/nu nude mice were purchased from Experimental Animal Center of Guangdong Province and used for the tumorigenicity assay. Briefly, $1 \times 10^{6}$ cells resuspended in RPMI- 1640 were injected into each mouse scapula. After 4 weeks, mice were sacrificed and xenograft tumors were removed, photographed, and weighed.
RNA sequencing. High-quality total RNA was extracted with TRIzol (Invitrogen), and polyA ${ }^{+}$mRNA was selected with oligo(dT) magnetic beads and chemically fragmented. RNA fragments were reverse transcribed to cDNA and the $\sim 200 \mathrm{bp}$ cDNA fraction was selected to construct a sequencing library. RNA-Seq was carried out on a HiSeq 2000 platform according to Illumina's protocol. After filtration, clean reads were aligned to the human reference genome (hg19) with Tophat2 (21). Single nucleotide variants were assessed with GATK2 (22). SnpSift was used for variant functional annotation (23).

Analysis of RNA editing with direct-sequencing. Site-specific RNA editing was measured with RT-PCR and direct sequencing (24). Editing was calculated with ImageJ software (http://rsb. info.nih.gov/ij/). Primers for PCR include FLNA-forward (CTTTGTTCCCGCTGAGATGG), FLNA-reverse (TTCCAG ACCTGCTCCGTAAG), FLNB-forward (GAAGAACTCAC ACTGCGTCC), FLNB-reverse (CCTGCTCGGGTGGTGTT AAT), COPA-forward (GCGAGGCATTGACTTCCATA), COPA-reverse (GGTCTCTGCGGAAAGTCTGA), IGFBP7forward (CTGCCCCTCTCCTCTTCCT), IGFBP7-reverse (GGGATTCCGATGACCTCACA).

Western blotting. Western blotting was carried out according to published standard methods. Antibodies used included ADAR2 (Sigma, 1:1,000), IGFBP7 (Abcam, 1:1,000), Matriptase (GeneTex, 1:1000), pAkt (Ser473) (CST, 1:1,000), total Akt (CST, 1:1,000), pBAD (SAB, 1:1,000), BAD (CST, 1:1,000), GAPDH (CST, 1:1,000), His-tag (CST, 1:1000), c-Myc-tag (MBL, 1:1,000).

Clinical samples. Tissue microarray (TMA) blocks with 116 ESCC samples, and three pairs of frozen ESCC primary tumor tissues and adjacent normal esophageal tissues were obtained from the Biobank of SYSUCC. Tissue samples were surgically removed between 2011 and 2014. Each participant signed an informed consent form and the study was approved by the Human Ethics Committee.

Immunohistochemical staining and assessment. Immunohistochemical staining for pAkt (antibody was purchased from CST) and pBAD (antibody was purchased from SAB) was conducted as previously reported (25). Two investigators scored immunoreactivity independently, and staining was scored as follows: i) percent positively staining cells: zero (0-10\%), 1 (11-25\%), 2 (26-50\%), 3 (51-75\%), and 4 (76-100\%); and ii) staining intensity: zero (no signal), 1 (weak), 2 (moderate), and 3 (strong). Staining scores were multiplied by percent scores for a final score (range 0-12).

Statistical analysis. Unless otherwise specified, data are means \pm SD of three experiments. SPSS (version 16.0) software was used for data analysis. $\mathrm{P}<0.05$ was considered statistically significant.

\section{Results}

ADAR2 functions as a tumor suppressor during ESCC progression. Previously, we reported that ADAR2 expression was downregulated in ESCC compared with adjacent 

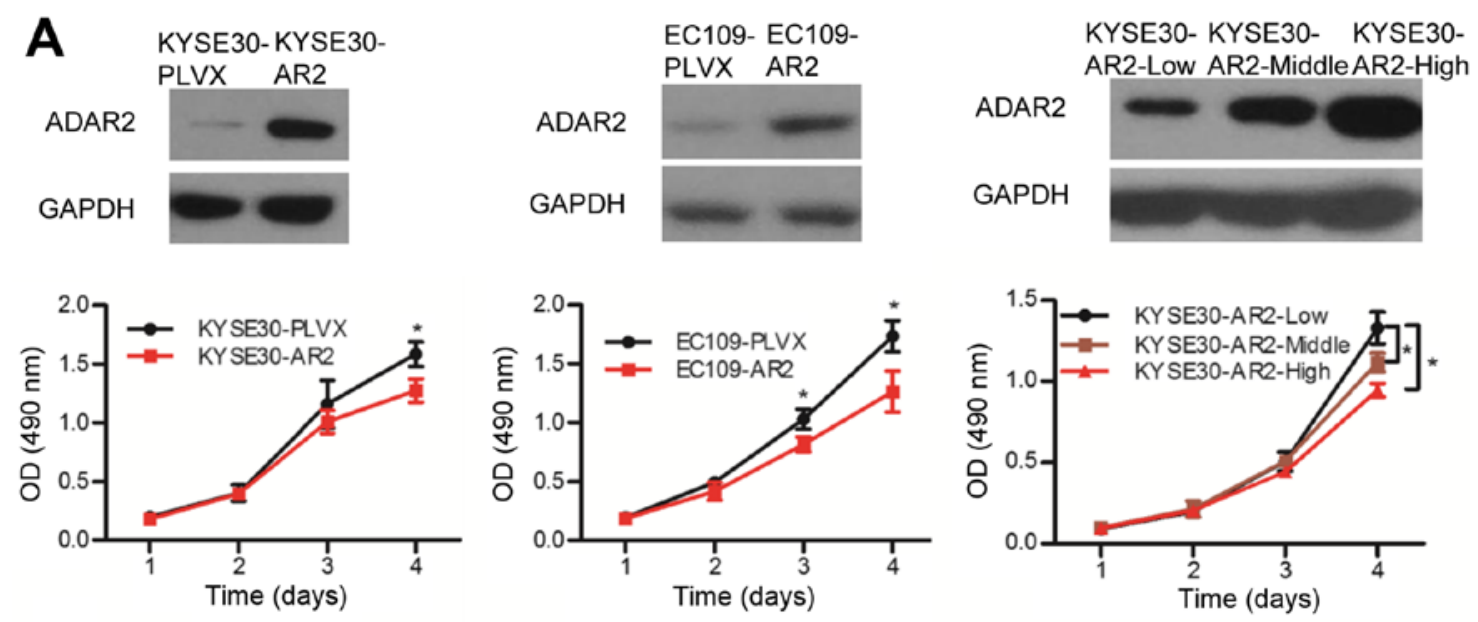

B
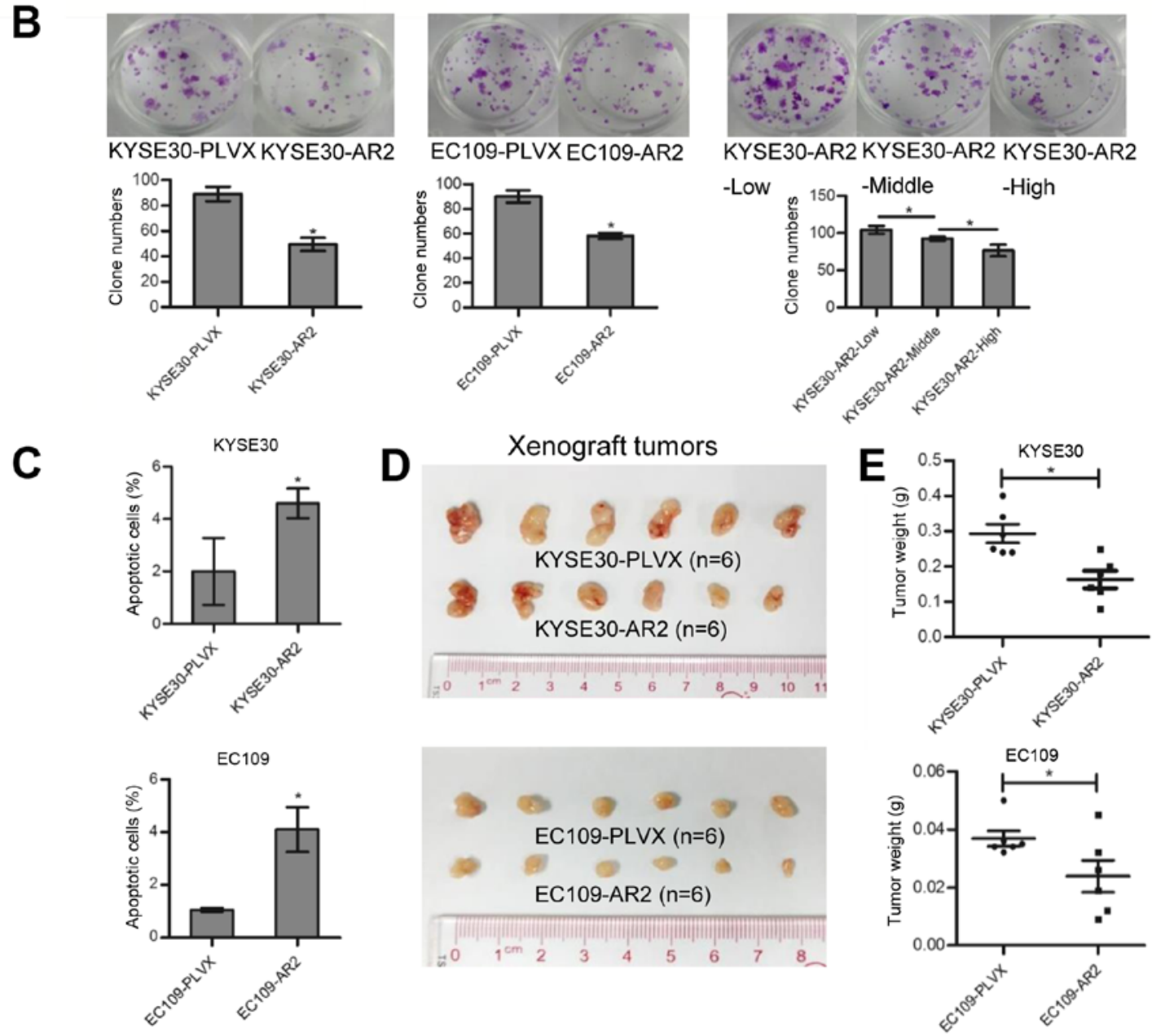

Figure 1. ADAR2 overexpression inhibits tumor growth and promotes ESCC cell apoptosis. (A) MTT assays were performed in ESCC cell lines as depicted in Materials and methods. Left, overexpression of ADAR2 in KYSE30; middle, overexpression of ADAR2 in EC109; right, dose-dependent effect of ADAR2 in KYSE30. KYSE30-AR2 and EC109-AR2, two ADAR2 stable transfection ESCC cells mediated by lenti-virus; KYSE30-PLVX and EC109-PLVX, two control cells infected with empty virus. KYSE30-AR2-Low/Middle/High cells had different ADAR2 expression and were derived from KYSE30-AR2. Upregulation of ADAR2 confirmed with western blotting of cell lysate. (B) Representative images and quantification of colon formation assay. (C) Data from Annexin V-FITC and PI staining. Early and late apoptosis. (D) Images of xenograft tumors derived from nude mice. (E) Tumor weights. Middle line is mean value. Error bars represent mean $\pm \mathrm{SD}$ from at least three independent experiments; ${ }^{*} \mathrm{P}<0.05$.

normal tissues, and patients with less tumor ADAR2 expression had worse prognoses (3). Furthermore, we found that several known editing targets of ADAR2 were edited in normal esophageal tissue, but they were almost not edited in the seven ESCC cell lines (data not shown), which suggested that ADAR2 lost its function in ESCC. To investigate the
ADAR2 role during ESCC progression, we used ADAR2 lentivirus to overexpress ADAR2 in ESCC KYSE30 and EC109 cell lines. Overexpressing lines were KYSE30-AR2 and EC109-AR2, and the control lentiviral-infected cells were KYSE30-PLVX and EC109-PLVX. Upregulation of ADAR2 was confirmed with western blotting (Fig. 1A). In vitro studies 
A
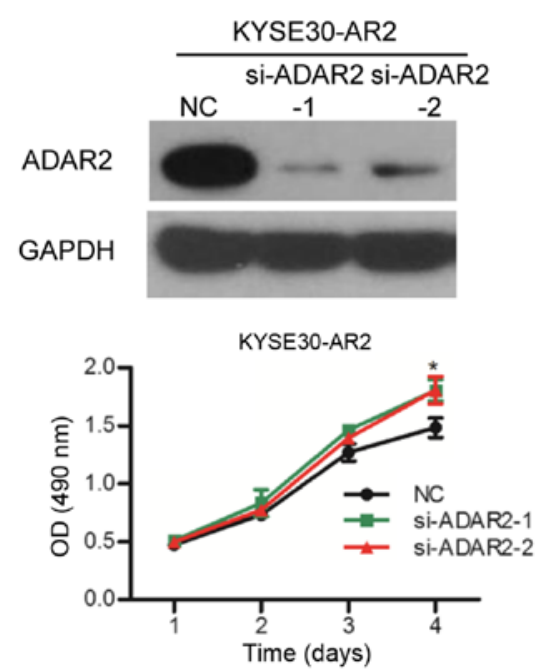

B

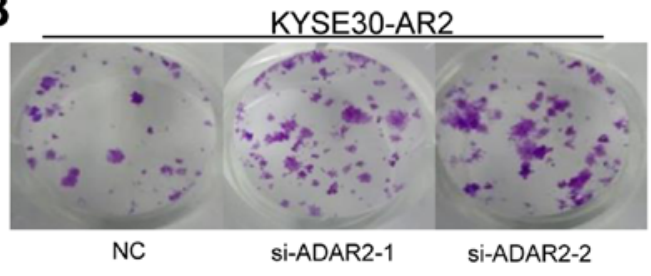

KYSE30-AR2

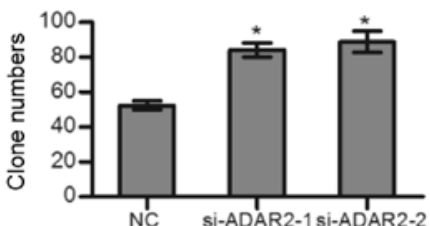

C

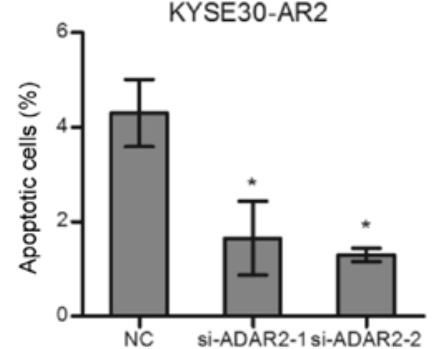

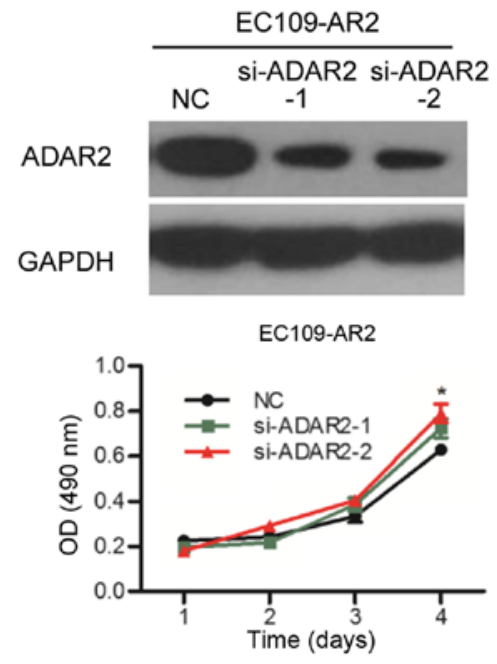

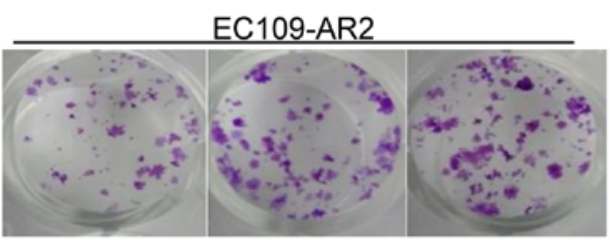

NC

Si-ADAR2-1 Si-ADAR2-2

EC109-AR2
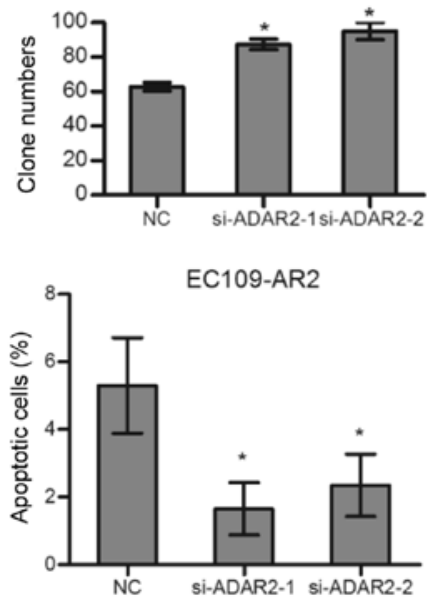

Figure 2. ADAR2 knockdown promotes cell growth and inhibits apoptosis in ESCCs. (A) MTT assay in ESCC cells. Left, knockdown of ADAR2 in KYSE30-AR2; right, knockdown of ADAR2 in EC109-AR2. si-ADAR2-1 and si-ADAR2-2, two different small interference RNAs of ADAR2; NC, siRNA negative control. Downregulation of ADAR2 was confirmed with western blotting of cell lysate. (B) Representative images and quantification of colon formation assay. (C) Results of Annexin V-FITC and PI staining. Early and late apoptosis. Error bars represent mean \pm SD from at least three independent experiments; ${ }^{\mathrm{P}} \mathrm{P}<0.05$.

confirmed that cells transfected with ADAR2 had less growth (Fig. 1A) and colony formation (Fig. 1B) compared to controls. Annexin $\mathrm{V}$ and PI staining revealed that overexpression of ADAR2 induced apoptosis (Fig. 1C). In contrast, knockdown of ADAR2 in KYSE30-AR2 and EC109-AR2 reversed cell growth and apoptosis effects to some extent (Fig. 2). Moreover, ADAR2 overexpression decreased tumor growth in xenograft tumor models (Fig. 1D and E).

Exogenous overexpression of ADAR 2 can edit the IGFBP7 transcript. To explore target genes edited by ADAR2 in ESCC, we used RNA-seq assay in KYSE30-PLVX and KYSE30-AR2 cells. We noted 19 A-to-G single nucleotide variation sites,
$\mathrm{G}$ fractions of which were $\geq 10 \%$ higher in KYSE30-AR2 than in KYSE30-PLVX cells. These were selected as potential A-to-I editing sites catalyzed by ADAR2. We validated these sites by assaying corresponding sequences of DNA and mRNA in KYSE30-AR2 and KYSE30-PLVX cells with direct sequencing. Finally, transcripts of four genes were identified as substrates of ADAR2 in ESCCs, including IGFBP7, FLNB, COPA, and FLNA (Fig. 3A). IGFBP7 was the only one reported to be associated with apoptosis (26-29), so we focused on IGFBP7. The editing site in IGFBP7 identified with RNA-seq was located at position 284 of the coding sequence, changing codon 95 from AAG (lysine) to AIG (arginine), henceforth termed K95R. K95R was validated in EC109-AR2 


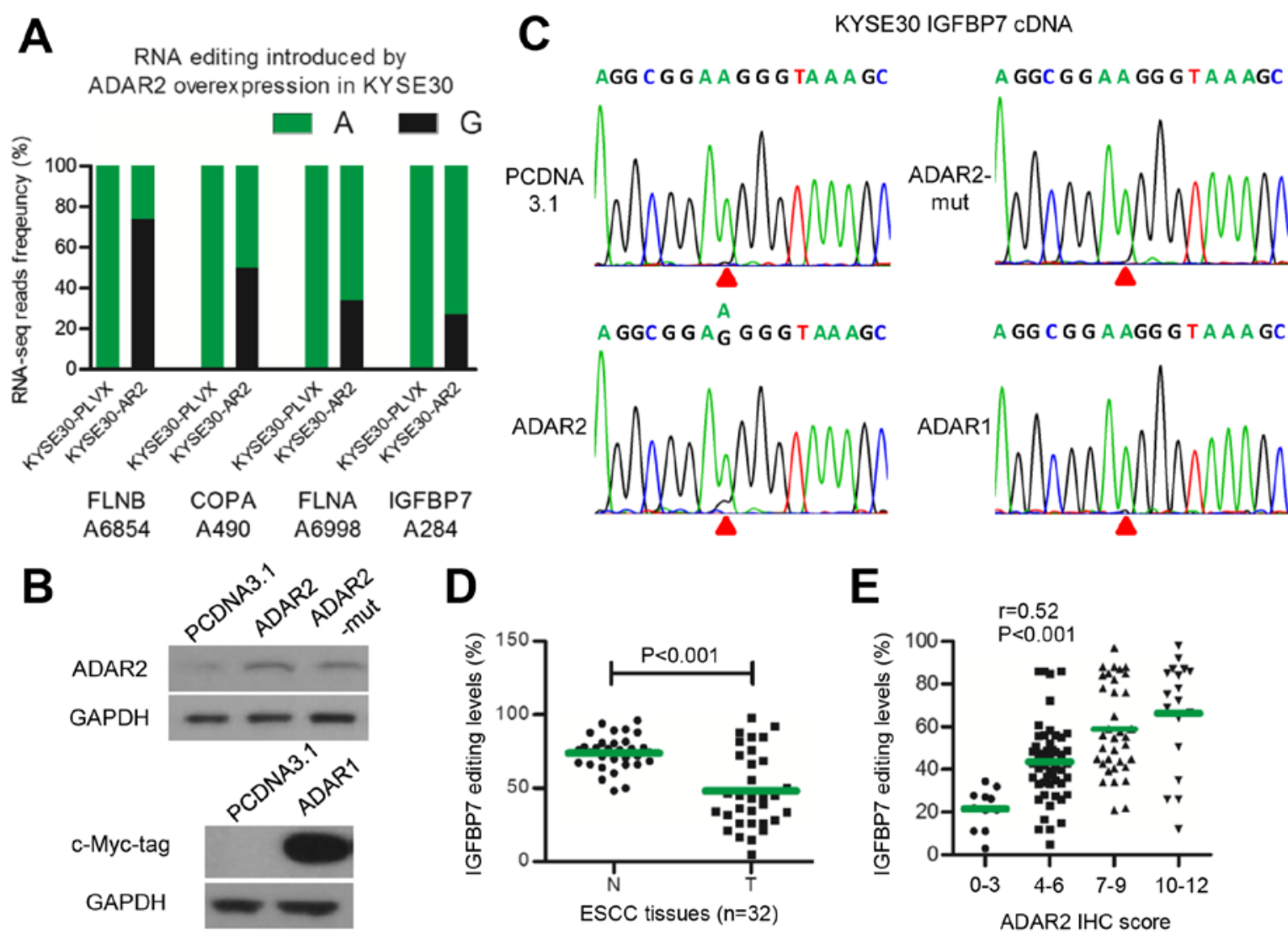

Figure 3. The editing targets of ADAR2 in ESCC. (A) RNA-seq revealed A-to-I editing sites of ADAR2 in KYSE30-AR2: FLNB A6854, COPA A490, FLNA A6998, and IGFBP7 A284. A, adenosine; the number followed, the nucleotide position in CDS. Degree of editing equals RNA-seq read frequency of G. (B) ADAR2 and ADAR1 protein were measured using western blotting $48 \mathrm{~h}$ after transfection of indicated vectors in KYSE30 cells. ADAR2-mut, an inactive form of ADAR2 containing a point mutation in the deaminase domain with GAPDH internal control. (C) Sequencing chromatographs of IGFBP7 cDNA measured $48 \mathrm{~h}$ after transfection of indicated vectors in KYSE30 cells. Red triangle directs the editing site. (D) Editing of IGFBP7 was quantified with direct sequencing in ESCC tumor and adjacent normal tissues. Midline is mean. (E) Correlation between ADAR2 protein and IGFBP7 editing in 116 ESCC tumors. Midline is mean; $r$ is Pearson correlation coefficient.

successfully (data not shown). We constructed and transfected three plasmids expressing wt ADAR1 and ADAR2, and RNA editing function loss type ADAR2 (ADAR2-mut) caused by deaminase domain mutation (30), respectively (Fig. 3B). Direct sequencing showed that only wt ADAR2 could edit IGFBP7 K95R, and neither ADAR1 nor ADAR2-mut could (Fig. 3C). We then measured IGFBP7 editing in 32 pairs of ESCC tumors and adjacent normal tissues. The data show that, similar to ADAR2 protein, IGFBP7 K95R editing decreased in tumors (Fig. 3D). Moreover, IGFBP7 K95R editing was moderately correlated with ADAR2 expression in 116 ESCC tumors $(r=0.52, \mathrm{P}<0.001)$ (Fig. 3E). Thus, these results suggested that IGFBP7 K95R is an ADAR2-specific editing target in ESCCs.

ADAR2 promotes ESCC apoptosis depending on IGFBP7 editing. IGFBP7 was previously reported to be an apoptotic promoter in several other cancers (26-29). So, we investigated the role of IGFBP7 in ADAR2-mediated apoptosis in ESCCs. At $48 \mathrm{~h}$ after transfection of IGFBP7 siRNAs and the siRNA negative control (nc) in KYSE30-AR2 and EC109-AR2, cells were harvested and apoptosis was assayed. Data show that knockdown of IGFBP7 abolished ADAR2-induced apoptosis (Fig. 4A). Transfection of ADAR2-mut, the RNA editing function loss type of ADAR2, did not induce apoptosis as wt did (Fig. 4B). Thus, the pro-apoptotic ability of ADAR2 might depend on IGFBP7 editing. However, overexpression of wt IGFBP7 (IGFBP7-wt) could promote ESCC apoptosis as well as the edited type (IGFBP7-K95R) (Fig. 4C and D). We conjectured that RNA editing could fine tune IGFBP7 function by affecting its RNA or protein stability, however, this fine-tuning was trivial when cells expressed exogenous IGFBP7 abundantly.

RNA editing of K95R protects IGFBP7 from matriptase proteolysis. According to previous reports, IGFBP7 is a secreted protein and IGFBP7 K95R editing is located within a matriptase protease recognition site (PRS) and inhibited matriptase mediated proteolysis in test tubes (31). In our study, we validated this phenomenon in culture medium of ESCC cells. Matriptase and IGFBP7 (IGFBP7-wt and IGFBP7-K95R) expression vectors were co-transfected into KYSE30 and EC109 as described in Fig. 4E. Then, $48 \mathrm{~h}$ after transfection, media were collected and assayed with western blotting. Data show that IGFBP7-wt could be partially cleaved at the $27 \mathrm{kDa}$-terminus and the $8 \mathrm{kDa} \mathrm{N}$-terminus (only the intact and $27 \mathrm{kDaC}$-terminus was measured with western blotting) by overexpression of matriptase, whereas little IGFBP7-K95R was cleaved (Fig. 4E). Moreover, western blot- 
A

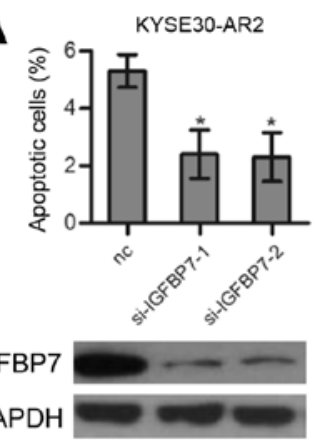

EC109-AR2

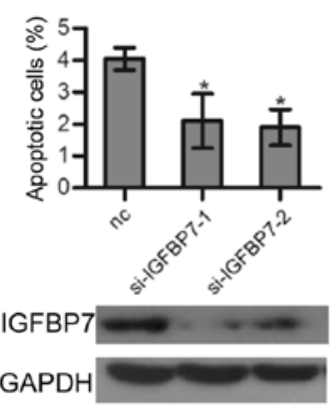

C

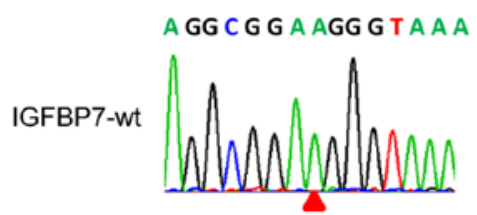

A GG C G G A GGG G T A A A

IGFBP7-K95R

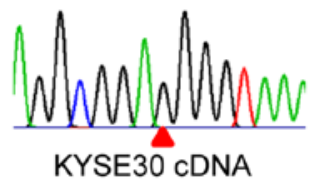

E
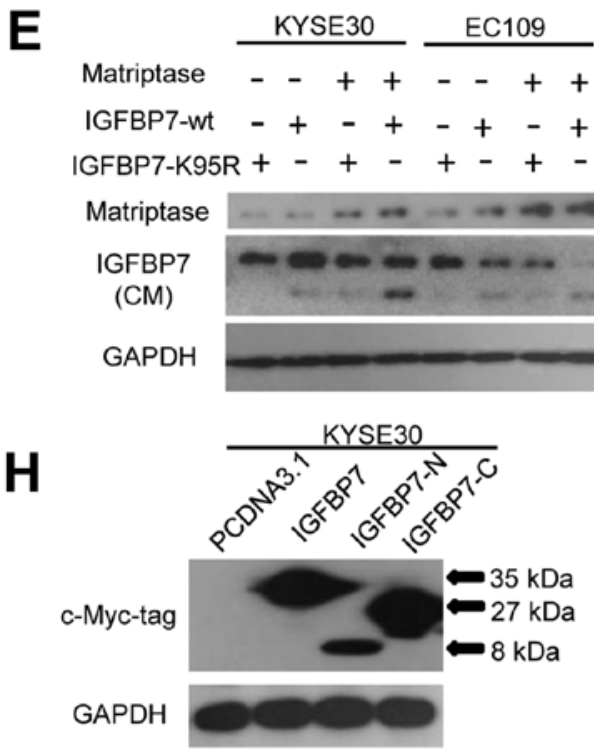

B
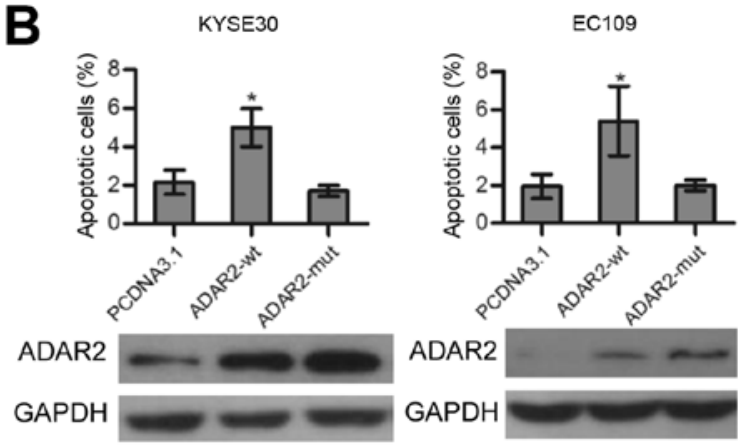

D
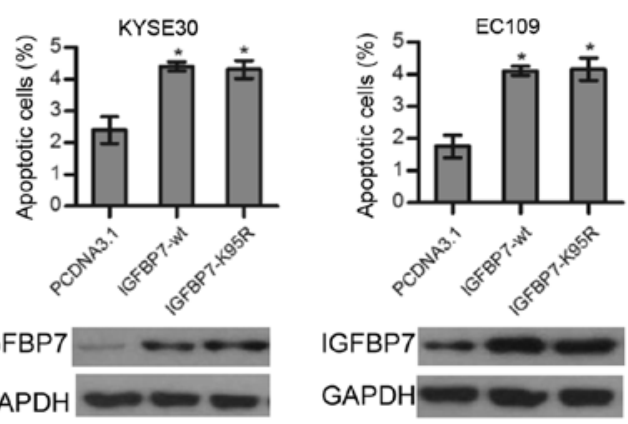

IGFBP7

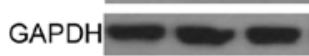

$\mathbf{F}$

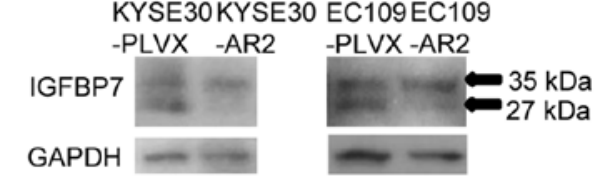

$\begin{array}{lllllllll}\mathbf{G} & 1 \mathrm{~N} & 1 \mathrm{~T} & 2 \mathrm{~N} & 2 \mathrm{~T} & 3 \mathrm{~N} & 3 \mathrm{~T}\end{array}$ $35 \mathrm{kDa}$
$27 \mathrm{kDa}$

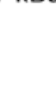
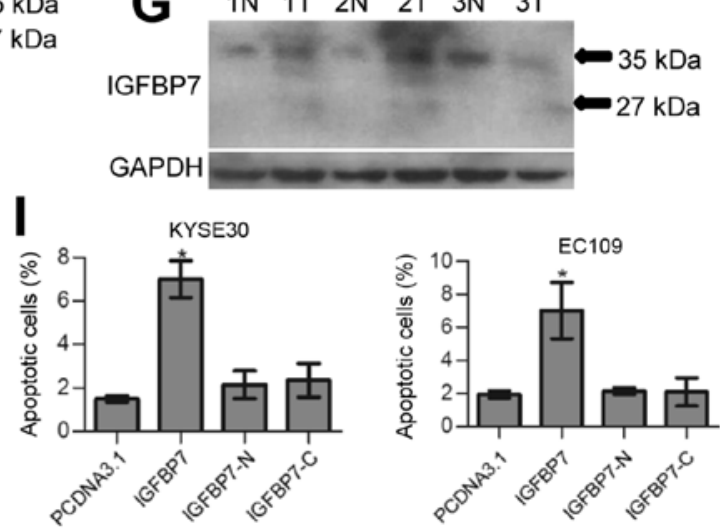

Figure 4. ADAR2 promotes apoptosis by editing and stabilizing IGFBP7. (A) Apoptosis and protein measured $48 \mathrm{~h}$ after IGFBP7 knockdown in KYSE30-AR2 and EC109-AR2. nc, siRNA negative control (B) Apoptosis and protein measured $48 \mathrm{~h}$ after overexpression of wt and editing function loss types of ADAR2 in KYSE30 and EC109. (C) Sequence chromatographs of IGFBP7 cDNA $48 \mathrm{~h}$ after transfection with indicated expression constructs in KYSE30. IGFBP7-wt is wild-type IGFBP7; IGFBP7-K95R is edited type of IGFBP7. (D) Apoptosis and protein measured $48 \mathrm{~h}$ after overexpression of wt and edited type of IGFBP7. (E) Edited type IGFBP7 was more stable than wt in culture medium. CM, culture medium. Intracellular GAPDH was a loading control. (F) Forms of IGFBP7 in xenograft tumors derived from indicated cell lines. (G) Forms of IGFBP7 in ESCC tumors and adjacent normal tissues. (H) Expression constructs were validated by western blotting $48 \mathrm{~h}$ after transfection in KYSE30. IGFBP7-N, the N-terminus of IGFBP7 (8 kDa); IGFBP7-C, the C-terminus of IGFBP7 (27 kDa). (I) Apoptosis measured $48 \mathrm{~h}$ after transfection of intact, $\mathrm{N}$-terminus or C-terminus of IGFBP7 in KYSE30 and EC109. Error bars represent mean \pm SD from at least three independent experiments; ${ }^{*} \mathrm{P}<0.05$.

ting showed that ADAR2 overexpressing xenograft tumor had less cleaved endogenous IGFBP7 (Fig. 4F). Thus, RNA editing of K95R can protect IGFBP7 against matriptase proteolysis in ESCC culture and xenografts. Measuring IGFBP7 expression in 3 pairs of surgically removed ESCC tumors and adjacent normal tissues revealed that more cleaved IGFBP7 was apparent in tumors (Fig. 4G), indicating a positive correlation between IGFBP7 editing and tissue stability.
Intact rather than cleaved IGFBP7 promotes cell apoptosis. Full-length IGFBP7, and not the truncated C-terminus, was reported to bind to IGF1R and inhibit downstream Akt signaling in mouse embryonic fibroblasts (MEFs) (17). We investigated the function of intact and cleaved IGFBP7 in ESCC cells with regard to apoptosis. The intact form, $\mathrm{N}$ - and $\mathrm{C}$-terminus of IGFBP7 expression constructs (each contained a BM40 signal peptide sequence in the vector) were transfected into ESCC 


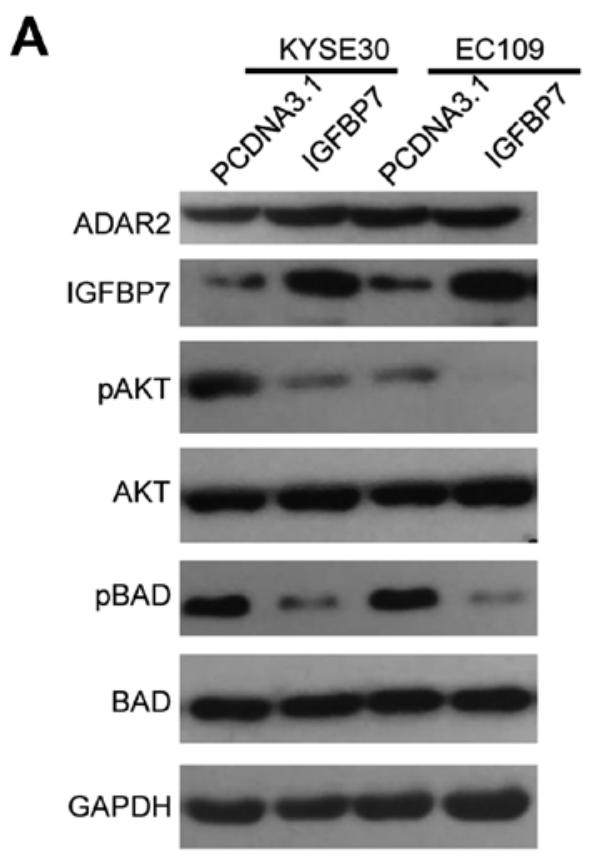

C

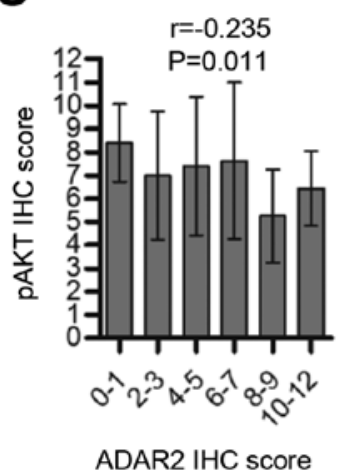

D

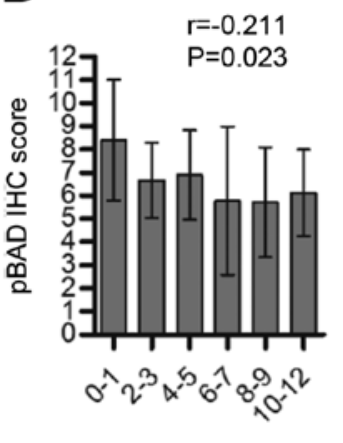

ADAR2 IHC score
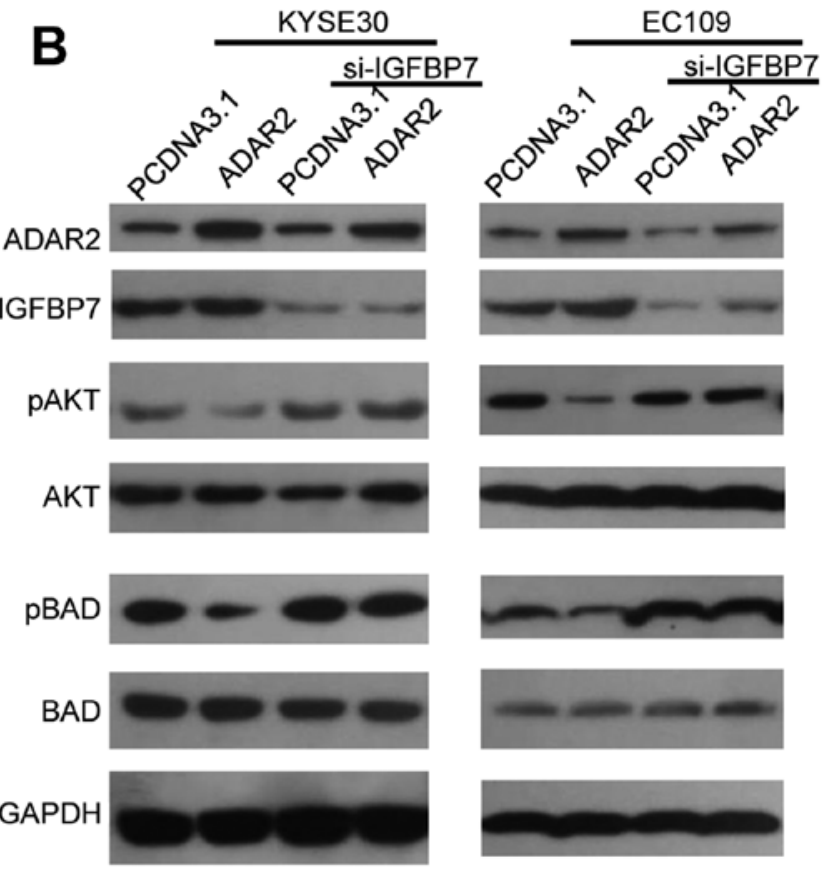

E
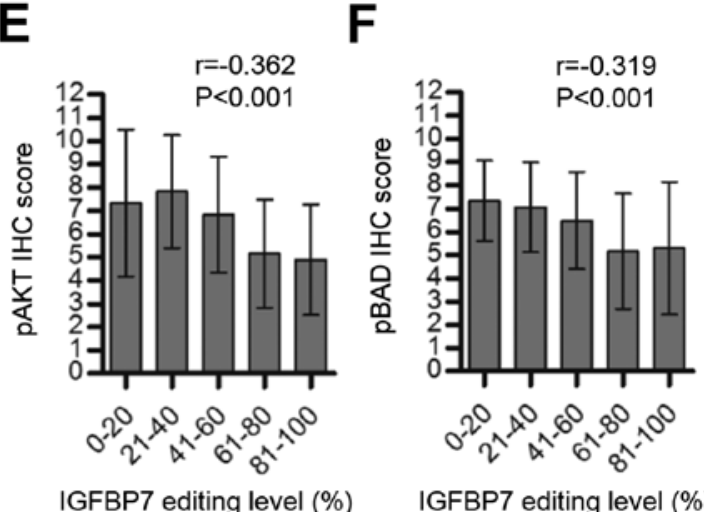

Figure 5. ADAR2 regulates Akt signaling depending on IGFBP7. (A) Inhibitory effect of IGFBP7 on Akt signaling in KYSE30 and EC109 cells. IGFBP7 and Akt signaling pathway proteins measured with western blotting $48 \mathrm{~h}$ after transfection of indicated expression constructs. (B) ADAR2 overexpression inhibited Akt signaling in KYSE30 and EC109 cells, and this depended on IGFBP7. ADAR2, IGFBP7, and Akt signaling pathway proteins measured via western blotting $48 \mathrm{~h}$ after co-transfection of indicated expression constructs and siRNA. Negative control siRNA was used to adjust background. (C-F) Correlations found in ESCC tumors of ADAR2 and pAkt (C), ADAR2 and pBAD (D), IGFBP7 editing and pAkt (E), and IGFBP7 editing and pBAD (F). Error bars represent mean $\pm S D ; r$ is the Pearson correlation coefficient; $n=116$.

cells separately. Only the intact form induced apoptosis $48 \mathrm{~h}$ after transfection (Fig. $4 \mathrm{H}$ and I). Thus, protein integrity is essential for IGFBP7 pro-apoptotic ability.

\section{ADAR2 inhibits Akt signaling depending on IGFBP7. IGFBP7} was reported to mediate apoptosis via regulating Akt signaling (17), so we validated this phenomenon in ESCC (Fig. 5A), and investigated whether ADAR2 could influence Akt signaling. As expected, ADAR2 overexpression in KYSE30 and EC109 cells inhibited phosphorylation of Akt and a downstream molecular BAD (BCL2 associated agonist of cell death) (Fig. 5B). However, when IGFBP7 was knocked down, this effect was abolished (Fig. 5B). Tissue arrays containing 116 ESCC tumors were immunohistochemically assayed for $\mathrm{pAkt}$ and pBAD, and data show that both were negatively correlated with ADAR2 (Fig. 5C and D) or IGFBP7 K95R editing (Fig. 5E and F) to a certain degree. Therefore, ADAR2 might protect BAD from phosphorylation and induce apoptosis by promoting IGFBP7-mediated Akt signaling inhibition.

\section{Discussion}

We report that ADAR2 promotes apoptosis by editing and stabilizing IGFBP7 in ESCC. This is in contrast to ADAR1, which acts as an oncogene in ESCC, and has a different editing profile (15). On the other hand, editing profiles of ADAR2 vary among different contexts. For example, in glial cells, ADAR2 can edit GluR-B, an AMPA receptor subunit, and recode Gln607 to Arg (Q/R). Decreased activity of ADAR2 leads to reduced editing at GluR-B $\mathrm{Q} / \mathrm{R}$ and promotes cell malignant transformation $(9,32,33)$. However, in ESCC, GluR-B expression was below the detection threshold for RT-PCR in our study (data not shown). Our findings strongly suggest that, in esophageal epithelia, the IGFBP7-IGF1R-Akt-BAD axis is a major 
ADAR2 target, which, when inhibited, leads to abnormal cell survival. These observations are consistent with previous findings that ESCC patients with tumors strongly expressing both IGF-II and IGF1R had worse survival rates (34), indicating that IGF1R might be crucial for ESCC therapy.

Until now, only a few editing sites of ADAR2 have been reported in neuronal tissues and beyond (14,35,36). More detailed editing information for ADAR2 must be gathered, especially for extraneuronal tissues. Here, we focused on editing targets of ADAR2 in ESCC. As we previously found, ADAR2 expression decreased in ESCC tumors compared to normal tissues, and the preliminary experiment showed that ADAR2 activity in ESCC cell lines were disrupted. Therefore, we overexpressed ADAR2 in KYSE30 cell line and RNAseq data revealed several editing targets of ADAR2. All four genes were previously reported as A-to-I RNA editing targets, and among them, FLNB, COPA, and FLNA were confirmed ADAR2 substrates. None of the four ADAR2 target genes has been correlated to ADAR2 function in any tissue type. One novelty of our data is that we were the first to report that ADAR2 was responsible for editing of IGFBP7 in ESCC and that ADAR2 regulates IGFBP7.

As previously reported, overexpression of IGFBP7 can promote apoptosis in cancers of the prostate (26), colorectum (27), thyroid (28), and breast (29). IGFBP7 could be silenced by promoter hyper-methylation in multiple neoplastic tissues (37-39). In this study, we report that ADAR2-mediated editing can protect IGFBP7 from cleavage in vitro and in vivo, and the integrity is essential for IGFBP7 to induce apoptosis in ESCCs. This suggests a novel regulatory mechanism for IGFBP7 in ESCCs. However, RNA editing may be a mild regulation mechanism of IGFBP7. We found that overexpression of $\mathrm{wt}$ IGFBP7 could promote ESCC apoptosis as did the edited type (Fig. 4C and D). Perhaps the abundance of exogenous IGFBP7 was beyond the regulating range of RNA editing.

BAD is a key regulator of programmed cell death, and its pro-apoptotic activity can be inhibited by phosphorylation regulated by Akt. Our results suggest that ADAR2 can inhibit Akt signaling, which, in turn, inhibits phosphorylation and releases pro-apoptotic activity of BAD. However, cancer cells might escape pro-apoptotic effects of ADAR2 by decreased transcription of IGFBP7, constitutive activation of AKT signaling due to RAS or PTEN mutations, EGFR or HER2 amplification, or INSR activation.

We did not study the effects of ADAR2 on endogenous IGFBP7 in a culture medium because it was below the threshold of detection by western blotting. However, we measured endogenous IGFBP7 in xenograft tumors and noted significantly less cleaved IGFBP7 in ADAR2 overexpressing xenograft tumors compared to control. More sensitive methods will be required in future studies.

Several recent whole-genome sequencing studies indicate that recurrent, somatic single-nucleotide variations are rare in ESCC (4-7). A-to-I editing reduction of IGFBP7 K95R to cause single nucleotide changes, however, offers consistent alteration across ESCC patients. In this regard, this post-transcriptional change might be more essential for cell transformation. On the other hand, distinct to genomic variations, RNA editing is a variation that can be spatiotemporally controlled by ADARs, permitting the design of target therapies to eliminate these variations. Our previous study indicated that miR-4707-5p aberrant overexpression in ESCC reduced ADAR2 protein, and patients with low ADAR2-expressing tumors had worse prognoses. Therefore, the miR-4707/ADAR2/IGFBP7 axis may be a useful target for ESCC treatment. Overall, we offer evidence that ADAR2 promotes apoptosis in ESCC by editing and protecting IGFBP7 from cleavage, and these data suggest that ADAR2 may have substantial efficacy for treating ESCC with minimal, if any, effects on normal tissues.

\section{Acknowledgements}

We thank Professor Xin-Yuan Guan of Sun Yat-sen University Cancer Center for the ESCC cell line. We also thank the Bank of Tumor Resources, Sun Yat-sen University Cancer Center for providing ESCC samples for this study. This study was supported by the grants from the National Natural Science Foundation of China (grant no. 81502056), the National Science Fund for Distinguished Young Scholars of China (grant no. 81325018) and the Key Project for International Cooperation and Exchange of the National Natural Science Foundation of China (grant no. 81220108022).

\section{References}

1. Pickens A and Orringer MB: Geographical distribution and racial disparity in esophageal cancer. Ann Thorac Surg 76: S1367-S1369, 2003.

2. Enzinger PC and Mayer RJ: Esophageal cancer. N Engl J Med 349: 2241-2252, 2003.

3. Qin HD, Liao XY, Chen YB, Huang SY, Xue WQ, Li FF, Ge XS Liu DQ, Cai Q, Long J, et al: Genomic characterization of esophageal squamous cell carcinoma reveals critical genes underlying tumorigenesis and poor prognosis. Am J Hum Genet 98: 709-727, 2016.

4. Song Y, Li L, Ou Y, Gao Z, Li E, Li X, Zhang W, Wang J, Xu L, Zhou Y, et al: Identification of genomic alterations in oesophageal squamous cell cancer. Nature 509: 91-95, 2014.

5. Gao YB, Chen ZL, Li JG, Hu XD, Shi XJ, Sun ZM, Zhang F, Zhao ZR, Li ZT, Liu ZY, et al: Genetic landscape of esophageal squamous cell carcinoma. Nat Genet 46: 1097-1102, 2014.

6. Zhang L, Zhou Y, Cheng C, Cui H, Cheng L, Kong P, Wang J, Li Y, Chen W, Song B, et al: Genomic analyses reveal mutational signatures and frequently altered genes in esophageal squamous cell carcinoma. Am J Hum Genet 96: 597-611, 2015.

7. Lin DC, Hao JJ, Nagata Y, Xu L, Shang L, Meng X, Sato Y, Okuno Y, Varela AM, Ding LW, et al: Genomic and molecular characterization of esophageal squamous cell carcinoma. Nat Genet 46: 467-473, 2014.

8. Nishikura K: Functions and regulation of RNA editing by ADAR deaminases. Annu Rev Biochem 79: 321-349, 2010.

9. Maas S, Patt S, Schrey M and Rich A: Underediting of glutamate receptor GluR-B mRNA in malignant gliomas. Proc Natl Acad Sci USA 98: 14687-14692, 2001.

10. Hogg M, Paro S, Keegan LP and O'Connell MA: RNA editing by mammalian ADARs. Adv Genet 73: 87-120, 2011.

11. Shah SP, Morin RD, Khattra J, Prentice L, Pugh T, Burleigh A, Delaney A, Gelmon K, Guliany R, Senz J, et al: Mutational evolution in a lobular breast tumour profiled at single nucleotide resolution. Nature 461: 809-813, 2009.

12. Chen L, Li Y, Lin CH, Chan TH, Chow RK, Song Y, Liu M, Yuan YF, Fu L, Kong KL, et al: Recoding RNA editing of AZIN1 predisposes to hepatocellular carcinoma. Nat Med 19: 209-216, 2013.

13. Jiang Q, Crews LA, Barrett CL, Chun HJ, Court AC, Isquith JM, Zipeto MA, Goff DJ, Minden M, Sadarangani A, et al: ADAR1 promotes malignant progenitor reprogramming in chronic myeloid leukemia. Proc Natl Acad Sci USA 110: 1041-1046, 2013

14. Chan TH, Lin CH, Qi L, Fei J, Li Y, Yong KJ, Liu M, Song Y, Chow RK, Ng VH, et al: A disrupted RNA editing balance mediated by ADARs (Adenosine DeAminases that act on RNA) in human hepatocellular carcinoma. Gut 63: 832-843, 2014. 
15. Qin YR, Qiao JJ, Chan TH, Zhu YH, Li FF, Liu H, Fei J, Li Y, Guan XY and Chen L: Adenosine-to-inosine RNA editing mediated by ADARs in esophageal squamous cell carcinoma. Cancer Res 74: 840-851, 2014

16. Galeano F, Rossetti C, Tomaselli S, Cifaldi L, Lezzerini M, Pezzullo M, Boldrini R, Massimi L, Di Rocco CM, Locatelli F, et al: ADAR2-editing activity inhibits glioblastoma growth through the modulation of the CDC14B/Skp2/p21/p27 axis Oncogene 32: 998-1009, 2013

17. Evdokimova V, Tognon CE, Benatar T, Yang W, Krutikov K, Pollak M, Sorensen PH and Seth A: IGFBP7 binds to the IGF-1 receptor and blocks its activation by insulin-like growth factors. Sci Signal 5: ra92, 2012.

18. Gommans WM, Tatalias NE, Sie CP, Dupuis D, Vendetti N, Smith L, Kaushal R and Maas S: Screening of human SNP database identifies recoding sites of A-to-I RNA editing. RNA 14: 2074-2085, 2008.

19. Levanon EY, Hallegger M, Kinar Y, Shemesh R, DjinovicCarugo K, Rechavi G, Jantsch MF and Eisenberg E: Evolutionarily conserved human targets of adenosine to inosine RNA editing. Nucleic Acids Res 33: 1162-1168, 2005.

20. Qi F, Cai P, Liu X, Peng M and Si G: Adenovirus-mediated P311 inhibits TGF- $\beta 1$-induced epithelial-mesenchymal transition in NRK-52E cells via TGF- $\beta 1-$ Smad-ILK pathway. Biosci Trends 9: 299-306, 2015.

21. Kim D, Pertea G, Trapnell C, Pimentel H, Kelley R and Salzberg SL: TopHat2: Accurate alignment of transcriptomes in the presence of insertions, deletions and gene fusions. Genome Biol 14: R36, 2013.

22. DePristo MA, Banks E, Poplin R, Garimella KV, Maguire JR, Hartl C, Philippakis AA, del Angel G, Rivas MA, Hanna M, et al: A framework for variation discovery and genotyping using next-generation DNA sequencing data. Nat Genet 43: 491-498, 2011.

23. Cingolani P, Patel VM, Coon M, Nguyen T, Land SJ, Ruden DM and Lu X: Using Drosophila melanogaster as a model for genotoxic chemical mutational studies with a new program, SnpSift. Front Genet 3: 35, 2012.

24. Li JB, Levanon EY, Yoon JK, Aach J, Xie B, Leproust E, Zhang K, Gao Y and Church GM: Genome-wide identification of human RNA editing sites by parallel DNA capturing and sequencing. Science 324: 1210-1213, 2009.

25. Liu DQ, Li FF, Zhang JB, Zhou TJ, Xue WQ, Zheng XH, Chen YB, Liao XY, Zhang L, Zhang SD, et al: Increased RIPK4 expression is associated with progression and poor prognosis in cervical squamous cell carcinoma patients. Sci Rep 5: 11955, 2015.

26. Mutaguchi K, Yasumoto H, Mita K, Matsubara A, Shiina H, Igawa M, Dahiya R and Usui T: Restoration of insulin-like growth factor binding protein-related protein 1 has a tumor-suppressive activity through induction of apoptosis in human prostate cancer. Cancer Res 63: 7717-7723, 2003.

27. Ruan W, Xu E, Xu F, Ma Y, Deng H, Huang Q, Lv B, Hu H, Lin J, Cui J, et al: IGFBP7 plays a potential tumor suppressor role in colorectal carcinogenesis. Cancer Biol Ther 6: 354-359, 2007.
28. Vizioli MG, Sensi M, Miranda C, Cleris L, Formelli F, Anania MC, Pierotti MA and Greco A: IGFBP7: An oncosuppressor gene in thyroid carcinogenesis. Oncogene 29: 3835-3844, 2010.

29. Benatar T, Yang W, Amemiya Y, Evdokimova V, Kahn H, Holloway $C$ and Seth A: IGFBP7 reduces breast tumor growth by induction of senescence and apoptosis pathways. Breast Cancer Res Treat 133: 563-573, 2012.

30. Cenci C, Barzotti R, Galeano F, Corbelli S, Rota R, Massimi L, Di Rocco C, O'Connell MA and Gallo A: Down-regulation of RNA editing in pediatric astrocytomas: ADAR2 editing activity inhibits cell migration and proliferation. J Biol Chem 283: 7251-7260, 2008.

31. Godfried Sie C, Hesler S, Maas S and Kuchka M: IGFBP7's susceptibility to proteolysis is altered by A-to-I RNA editing of its transcript. FEBS Lett 586: 2313-2317, 2012.

32. Ishiuchi S, Tsuzuki K, Yoshida Y, Yamada N, Hagimura N, Okado H, Miwa A, Kurihara H, Nakazato Y, Tamura M, et al: Blockage of $\mathrm{Ca}(2+)$-permeable AMPA receptors suppresses migration and induces apoptosis in human glioblastoma cells. Nat Med 8: 971-978, 2002.

33. Ishiuchi S, Yoshida Y, Sugawara K, Aihara M, Ohtani T, Watanabe T, Saito N, Tsuzuki K, Okado H, Miwa A, et al: $\mathrm{Ca}^{2+}$-permeable AMPA receptors regulate growth of human glioblastoma via Akt activation. J Neurosci 27: 7987-8001, 2007.

34. Imsumran A, Adachi Y, Yamamoto H, Li R, Wang Y, Min Y, Piao W, Nosho K, Arimura Y, Shinomura Y, et al: Insulin-like growth factor-I receptor as a marker for prognosis and a therapeutic target in human esophageal squamous cell carcinoma. Carcinogenesis 28: 947-956, 2007.

35. Riedmann EM, Schopoff S, Hartner JC and Jantsch MF: Specificity of ADAR-mediated RNA editing in newly identified targets. RNA 14: 1110-1118, 2008.

36. Stulić M and Jantsch MF: Spatio-temporal profiling of Filamin A RNA-editing reveals ADAR preferences and high editing levels outside neuronal tissues. RNA Biol 10: 1611-1617, 2013.

37. Kanemitsu N, Kato MV, Miki T, Komatsu S, Okazaki Y, Hayashizaki Y and Sakai T: Characterization of the promoter of the murine mac25 gene. Biochem Biophys Res Commun 279: 251-257, 2000.

38. Ahmed S, Yamamoto K, Sato Y, Ogawa T, Herrmann A, Higashi S and Miyazaki K: Proteolytic processing of IGFBPrelated protein-1 (TAF/angiomodulin/mac25) modulates its biological activity. Biochem Biophys Res Commun 310: 612-618, 2003.

39. Lin J, Lai M, Huang Q, Ma Y, Cui J and Ruan W: Methylation patterns of IGFBP7 in colon cancer cell lines are associated with levels of gene expression. J Pathol 212: 83-90, 2007. 